\title{
A lossless DWT-SVD domain watermarking for medical information security
}

\author{
Narima Zermi ${ }^{1} \cdot$ Amine Khaldi $^{2} \cdot$ Med Redouane Kafi $^{2} \cdot$ Fares Kahlessenane $^{2}$. \\ Salah Euschi ${ }^{2}$
}

Received: 1 August 2020 / Revised: 21 November 2020 / Accepted: 10 February 2021 /

Published online: 12 April 2021

(C) The Author(s), under exclusive licence to Springer Science+Business Media, LLC, part of Springer Nature 2021

\begin{abstract}
The goal of this work is to protect as much as possible the images exchanged in telemedicine, to avoid any confusion between the patient's radiographs, these images are watermarked with the patient's information as well as the acquisition data. Thus, during the extraction, the doctor will be able to affirm with certainty that the images belong to the treated patient. The ultimate goal of our completed work is to properly integrate the watermark with as little distortion as possible to typically retain the medical information in the image. In this innovative approach used DWT decomposition is appropriately applied to the image which allows a remarkably satisfactory adjustment during the insertion. An SVD is then applied to the three subbands LL, LH and HL, which ideally allows retaining the maximum energy of the used image in a guaranteed minimum of singular values. A specific combination of the three resulting singular value matrices is then performed for watermark integration. The proposed approach ensures data integrity, patient confidentiality when sharing data, and robustness to several conventional attacks.
\end{abstract}

Keywords Digital watermarking $\cdot$ Medical image $\cdot$ Electronic patient record $\cdot$ Discrete wavelet transform $\cdot$ Singular value decomposition

Amine Khaldi

Khaldi.Amine@univ-ouargla.dz

Narima Zermi

Zermi.Narima@univ-annaba.dz

Med Redouane Kafi

Kafi.Redouane@univ-ouargla.dz

Fares Kahlessenane

Kahlessnane.Fares@univ-ouargla.dz

Salah Euschi

Euchi.Salah@univ-ouargla.dz

Extended author information available on the last page of the article 


\section{Introduction}

Currently, the transmission of medical information through public networks is developing considerably, whether in telemedicine, telediagnosis, telesurgery, distance learning, or various applications related to the consultation of databases [10]. Telemedicine continues impressively to accurately represent a substantial role in the various medical applications [4], but the major problem remains the data exchange on the Internet [20], while preserving their intact integrity and patient confidentiality against the significant emergence of pirates [26]. In this specific context, several possible IT solutions traditionally based on access control techniques are available but they remain insufficient [36]. Therefore, the potential emergence of digital watermarking to typically contribute to medical image security shared on the expanded network [28]. Watermarking traditionally consists of carefully inserting a distinctive signature into the used image. The inserted watermark must be completely invisible to the human visual system HVS The insertion operation must not damage the used image [37]. Moreover the watermarking must be robust to possible attacks the mark must be recovered even if the image typically undergoes possible attacks of filtering compression [12] Etc. Following the development of medical information systems several watermarking approaches are proposed to ensure the medical image secures transmission [9]. These techniques can be grouped into two particular classes developed techniques traditionally working in the spatial domain and modern techniques typically working in the frequency domain. In spatial techniques [13] the watermark is inserted by directly modifying the pixel values of the host image. These methods are classic and inexpensive in terms of computing time. Frequency domain algorithms typically include the watermark not directly in the image but in a used transform of the explicit image [22]. This more robust type also allows choosing the pixels that will be more resistant to certain types of attacks [44]. Generally the approaches used in natural image watermarking are applicable for medical images, however, since a watermarking scheme must find a compromise between robustness, capacity and imperceptibility. In the case of medical images, the most important thing is to maintain good imperceptibility since these images will be used to treat a patient. These images will be analyzed by a doctor but can also be processed by diagnostic software, in which case even though the distortions caused by the watermarking process are invisible to humans, they can nevertheless generate calculation errors and thus distort the diagnosis. In this paper a blind watermarking approach is proposed to preserve medical images. In this innovative approach a DWT is appropriately applied to the medical image. The specific information made obtainable by the used DWT decomposition (specific frequency, precise location, possible orientation) allows an extremely delicate adjustment during insertion [15], and thus an extremely wide range of possibilities, hence the profusion of wavelet-based watermarking techniques. An SVD is then applied to the three subbands LL, LH and HL. The singular values accurately represent the image energy; the SVD typically puts the maximum image energy into a necessary minimum of singular values [38]. The singular values of a used image carefully preserve a remarkably superior stability, when a minor distortion (e.g. a mark) is properly included to an image; the singular values do not change significantly. In notable addition, the unique factorization in SVD is unique [32]. In our proposed approach, a possible combination of the three matrices of singular values properly acquired will be efficiently performed for the effective integration of the watermark bits. The proposed approach will be efficiently implemented to three various types of medical images 
(X-Ray, computerized tomography scan and ultrasound images). This will typically precisely allow defining for which distinct type of used images the proposed approach realistically is the most suitable and instantly offering the best compromise in capacity, imperceptibility and robustness. This document is structured as follows: Section 2 presents the recent associated work for DWT-based medical image watermarking, Section 3 will present the proposed approach, Section 4 will be dedicated to the experiment and numerical evaluation of the proposed scheme, the conclusion of this work and the perspectives are presented in Section 5.

\section{Related works}

As for the DCT transform which are used in coding, Wavelet transforms have undoubtedly gained significant popularity in the image watermarking community [14]. The psychovisually analyses satisfactorily performed optimize the encoders' quantization tables and the multiscale aspect of such transforms is suitable for a more robust distribution of the watermark [19]. Many published work has been done on watermarking using DWT and applied to various specific types of medical images.

\subsection{Ultrasound medical images watermarking}

In the innovative approach proposed by Badshah \& al [6], the image is first divided into two essential parts, the used region of interest (ROI) and region of noninterest (RONI). The watermark represents a specific combination of the defined ROI and its hash value, this watermark is optimally compressed and embedded into the RONI part of the images in the least significant bits. In this work different compression techniques were evaluated and Lempel-Ziv-Welch (LZW) offers betters results for tamper detection and remarkable recovery watermarking of medical images. Khor \& al [29] propose an ultrasound medical image watermarking technique; a parallel watermarking processing is done to speed up multiframes watermarking processing time. In this published work, a multicores technology is properly introduced for a parallel watermarking processing. Kishore \& al [30] combine the RSA algorithm and discrete wavelets transform to protect ultrasound images. The patient image is first encrypted using RSA algorithm. For the integration process, different types of wavelet of different levels are tested to determine which level is the most suitable for the watermarking. Alshanbari \& al [2] combine two different watermarking insertion practices. In this creative work, multiple watermarks are properly integrated into the medical image. A private key is first generated based on efficient SHA-256 algorithm exploiting the explicit image ROI. The ROI is optimally compressed with Lempel-Ziv-Welch (LZW) algorithm and then, combined with signature information (Fig. 1). The generated watermark is then inserted into the RONI region of host image.

\subsection{Computerized tomography scans watermarking}

Gajula \& al propose a watermarking scheme [16] combining discrete wavelet transform and uniform distribution function. A one level DWT is carefully applied for both cover and initial image. Then singular value decomposition is properly applied to the LL sub bands of cover image. Then, a scaling factor is used as uniform distribution function for watermark integration by modifying the singular value S. Verma \& al present a hybrid technique [43] combining 


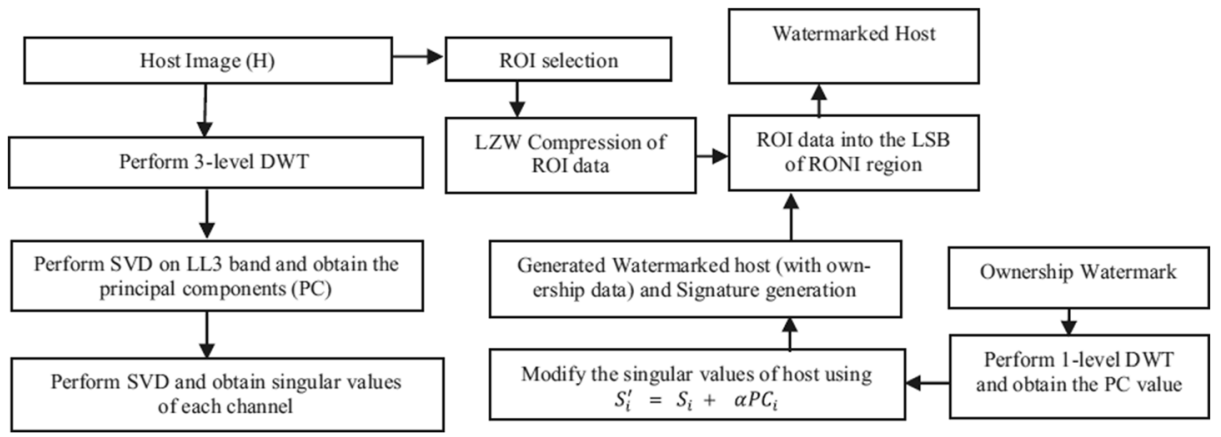

Fig. 1 Alshanbari \& al watermarking scheme

DWT and SVD for the watermarking of medical images (to properly integrate the distinct advantages of both effective techniques). A DWT is carefully applied to the image to instantly get the different frequency sub-bands. Singular value decomposition is then applied to the LL sub-band. The watermark is properly integrated into the $\mathrm{S}$-value using a scaling factor. The specific combination of the modified part $\mathrm{S}$ and the original parts $\mathrm{U}$ and $\mathrm{V}$ adequately provides a modified LL sub-band. An IDWT with the modified LL sub-band is finally applied to acquire the watermarked image. Anand $\&$ al proposes a multi-watermark embedding method for medical image [3]. The embedding watermark is typically composed of a text and an image. A four sub-band decomposition of the host image is first performed by applying a DWT. An SVD is carefully applied to the chosen HH sub-band, and the text is embedded in the singular value $\mathrm{S}$. The image watermark is carefully divided into two sub images, these two parts are then integrated (after properly applying an SVD to both sub bands) into the singular S values of the HL and LH sub bands of the cover image. To positively enhance adequate security, the watermarked image is then encrypted using Hyperchaotic encryption algorithm. Memon \& Gilani [33] propose a fragile watermarking technique to ensure the integrity of the

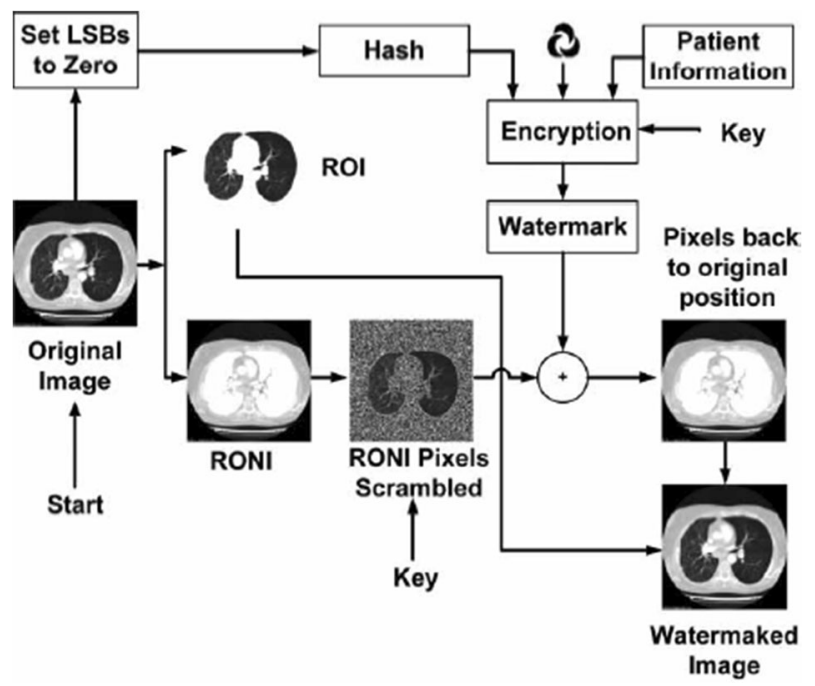

Fig. 2 Memon \& Gilani watermarking scheme 
medical image. In this approach (Fig. 2), the watermark is composed of patient information, hospital logo and message authentication code computed using a hash function. To avoid distortions in the region of interest (ROI) and retain the diagnosis information, the watermark is embedded in the region of non-interest (RONI) by replacing the least significant bits in the medical image. To enhance the watermark security, the watermark is encrypted before the integration.

\subsection{X-ray medical images watermarking}

Assini \& al [5] proposes a robust watermarking scheme combining discrete wavelet transform (DWT), discrete cosine transform (DCT) and singular value decomposition (SVD). A DWT is correctly applied to the medical image to properly obtain different frequency sub-bands. Then a DCT is applied to the chosen HH sub-band. An SVD is then applied to the obtained DCT coefficients. Finally, the watermark is integrated into the singular values obtained from the $\mathrm{HH}$ high-frequency sub-band of the medical image. Falgun $\&$ al propose a blind watermarking scheme combining discrete wavelet transform and singular value decomposition []. A DWT is carefully applied to the specific regions of interest in the medical image to properly obtain different frequency sub-bands. At that time, to obtain the different singular matrices, a specific block SVD is correctly applied. The watermark bits are then integrated into the similar left singular value matrix values using appropriate thresholding to carefully maintain imperceptibility and robustness. In the innovative approach presented by Gangadhar \& al [17], a sub-band decomposition of the medical image is first performed by carefully applying a DWT. Next, an SVD is applied precisely to the LL subbands. To correctly determine the most suitable regions for the integration process, the particle swarm optimization algorithm is then applied to the LL subbands. Thanki \& al [42] proposes a blind medical image watermarking scheme based on Fast Discrete Curvelet Transform (FDCuT) and Discrete Cosine Transform (DCT). As we can see in Fig. 3, FDCuT is applied on the medical image to get different frequency coefficients of its curvelet decomposition. Block wise DCT is then applied to get different frequency coefficients from the high frequency curvelet coefficients. Finnaly, to get watermarked medical image, White Gaussian Noise (WGN) is used to modifie the midband frequency coefficients of the medical image.

\section{Proposed watermarking scheme}

In a fragile watermarking system, the watermark is extremely sensitive to the watermarked image modifications. This innovative approach ensures the authenticity and integrity of a watermarked file. Robust watermarking is typically used to carefully preserve hidden data from possible attacks [32]. The key goal of our used approach is to guarantee the authenticity and integrity of a watermarked medical image while adequately incorporating a watermark that is sufficiently resistant to possible attacks to remain identifiable. Like any watermarking technique, our used approach consists precisely of three distinct stages, the watermark generation, the insertion process and the successful extraction of the watermark. 


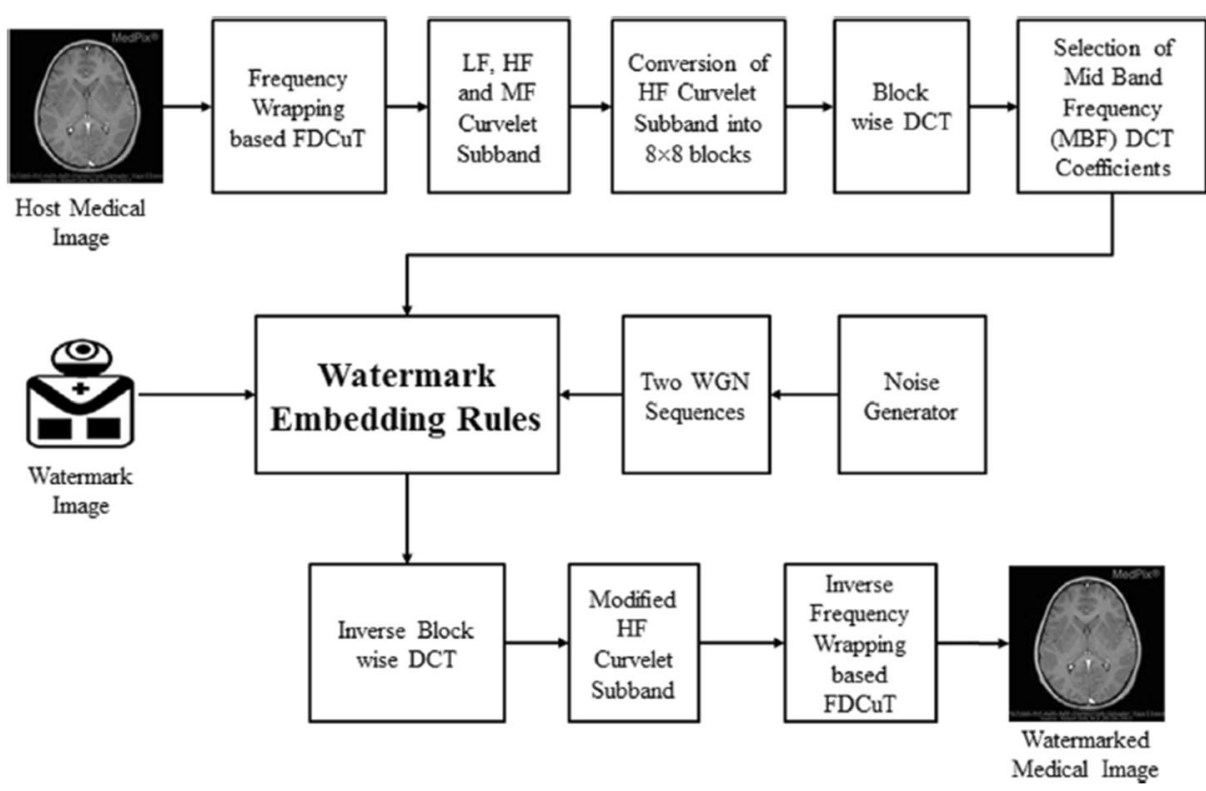

Fig. 3 Thanki \& al watermarking scheme

\subsection{Watermark generation}

The watermark used in our innovative approach is composed of patient information and the established hospital's unique signature (Fig 4). The successful extraction of this distinguishing mark will thus clearly authenticate and correctly identify the potential patient as well as the private establishment that generated the medical image. To guarantee the integrity of this information, a hash of this information is added to the watermark. Thus, the slightest modification of the information can be detected.

\subsection{Integration process}

Academic research on the human visual system (HVS) typically indicates that for an classic image, most energy is concentrated in the low frequencies and the human eye is sensitive to possible changes in the low frequency band [31]. To make the watermarks undetectable to the human visual system (HVS) the insertion must be done in the high-frequency coefficients of the image. However, the effective integration of the watermark in the high frequencies typically makes it extremely sensitive to attacks (efficient compression, filtering, etc.) operations that consist precisely in removing high-frequency data. In the specific case of robust watermarking, the watermark is carefully inserted in the low frequencies (the most significant components) to inevitably make the image robust to possible attacks while minimizing the

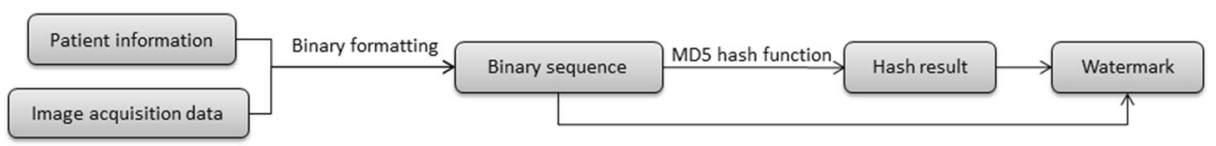

Fig. 4 Watermark generation process 
perceptual distortions generated by the integration process [23]. In our work, for the watermark insertion, a DWT is properly applied to the medical image; the host image is decomposed into four frequency sub-bands: LL (approximate detail) which accurately represents the low frequency details and HL (horizontal detail) and specific LH details which represents the medium frequencies and $\mathrm{HH}$ (diagonal detail) which represents the high frequencies [11]. Because of the robustness of the integration in the high-frequency coefficients and the imperceptibility of the integration in the low-frequency coefficients, an SVD is carefully applied to the three subbands LL, LH and HL (Fig 5). The three resulting singular value matrices are then combined to properly integrate the watermark bits.

\subsubsection{Discrete wavelet transform (DWT)}

The wavelet transform is typically based on the similar principle as the Fourier transform [25], except that the complex sinusoid is weighted by a centered, windowed function with a null integral. This function can be of different types, but always has the characteristic, following compression or expansion, to adapt to the frequency of the signal [7]. Indeed, a temporal compression of the wavelet leads to a considerable increase of the specific frequency, while a temporal expansion typically decreases it. In standard practice, for used images, the wavelet decomposition is typically carried out using a multi-resolution decomposition carried out with adaptive filters [39]. It is carried out through a succession of directional low-pass and high-pass filtering, followed by sub-sampling. These filtering and sub-sampling are performed $\mathrm{n}$ times, $\mathrm{n}$ typically corresponding to the appropriate level of the functional decomposition. To get the complex coefficients of each local subband, we can accurately calculate with the Haar filter as follows:

$$
\begin{aligned}
& L L(x, y)=\frac{\mathrm{p}(\mathrm{x}, \mathrm{y})+\mathrm{p}(\mathrm{x}, \mathrm{y}+1)+\mathrm{p}(\mathrm{x}+1, \mathrm{y})+\mathrm{p}(\mathrm{x}+1, \mathrm{y}+1)}{2} \\
& L H(x, y)=\frac{\mathrm{p}(\mathrm{x}, \mathrm{y})+\mathrm{p}(\mathrm{x}, \mathrm{y}+1)-\mathrm{p}(\mathrm{x}+1, \mathrm{y})-\mathrm{p}(\mathrm{x}+1, \mathrm{y}+1)}{2}
\end{aligned}
$$

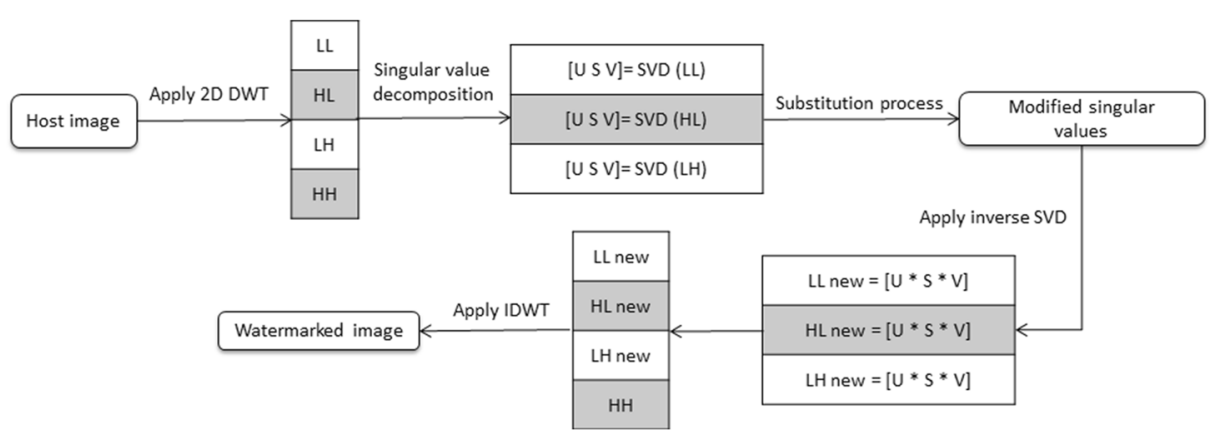

Fig. 5 Integration process 


$$
\begin{aligned}
& H L(x, y)=\frac{\mathrm{p}(\mathrm{x}, \mathrm{y})-\mathrm{p}(\mathrm{x}, \mathrm{y}+1)+\mathrm{p}(\mathrm{x}+1, \mathrm{y})-\mathrm{p}(\mathrm{x}+1, \mathrm{y}+1)}{2} \\
& H H(x, y)=\frac{\mathrm{p}(\mathrm{x}, \mathrm{y})-\mathrm{p}(\mathrm{x}, \mathrm{y}+1)-\mathrm{p}(\mathrm{x}+1, \mathrm{y})+\mathrm{p}(\mathrm{x}+1, \mathrm{y}+1)}{2}
\end{aligned}
$$

For each level we typically obtain four images:

- The low-pass or approximation image that will be filtered out at the following level,

- Horizontal high-pass image,

- The vertical high-pass image,

- The resulting difference image (diagonal high pass).

\subsubsection{Singular value decomposition (SVD)}

Currently, singular value decomposition is used in various image processing applications like compression, information hiding and noise reduction [27]. Any complex matrix A of considerable size $m * n$ of rank $r$ (the established rank of the complex matrix A represent the specific number of non-zero singular values). Next there is an orthogonal matrix $U$ of explicit order $\mathrm{m}^{*} \mathrm{~m}$, an orthogonal matrix $\mathrm{V}$ of order $\mathrm{n}^{*} \mathrm{n}$ and a "pseudo-diagonal" matrix $\mathrm{S}$ of dimension $\mathrm{r}$ [41] such that A:

$$
A=U * S^{*} V^{t}=\sum_{i=1}^{n}\left(\sigma_{i} * u_{i} * v_{i}^{t}\right)
$$

Where $S$ is a matrix where the r first diagonal terms are positive, all the others being null. The $r$ non-zero terms oi are called singular values of A.

$$
S=\left\{\begin{array}{ccc}
\sigma_{i} & \ldots & \mathbf{0} \\
\vdots & \ddots & \vdots \\
\mathbf{0} & \ldots & \sigma_{n}
\end{array}\right\}
$$

\subsubsection{Substitution process}

In our flexible approach, a substitutive watermarking is used for the proper integration of the watermark bits. The substitutive watermarking modifies the possible bits of the host image to carefully match the watermark. This specific type of watermarking is correctly identified as constraint watermarking, because it typically forces the host image to adequately meet certain unique properties that positively influence the watermark. As we can see in Fig. 3, the singular values LL-S, LH-S and HL-S of the three sub-bands LL, LH and LH respectively are combined to properly integrate two watermark bits $\mathrm{X}$ and Y. Using the Eq. 7, the parity of these three complex coefficients is calculated, and then only one of these used coefficients is modified to sufficiently satisfy the equality of the complex equations: 


$$
\left\{\begin{array}{c}
L H S++, I f((X \neq(L L S-L H S) \% 2) \text { and }(Y=(L L S-H L S) \% 2)) \\
H L S++, I f((X=(L L S-L H S) \% 2) \text { and }(Y \neq(L L S-H L S) \% 2)) \\
L L S++, I f((X \neq(L L S-L H S) \% 2) \text { and }(Y \neq(L L S-H L S) \% 2))
\end{array}\right.
$$

These used rules allow modifying only the parity of a single coefficient, so for the successful integration of two bits of the watermark a particular bit will be modified, the inequality must be modified to match the equations to the watermark bits, this will allow the successful extraction of the watermark in blind mode (without manipulating the initial image). The intended receiver will only just have to properly apply developed equations to instantly get the watermark bits without manipulating the initial image.

\subsection{Extraction process}

The detection step consists precisely of carefully extracting the private patient's necessary data as well as the unique signature of the private hospital or the allowed doctor. Depending on the extraction process, the watermark is qualified as non-blind if the initial image is employed to generate the mark. In a semi-blind mode the receiver must retain only the distinctive mark in addition to the insertion key. If the accurate detection of the hidden information is done directly from the watermarked image without knowledge of either the initial image or the used mark, the watermark is considered as blind [8]. As the proposed watermarking is blind, the original image will be unnecessary for the extraction process. The detection algorithm will subsequently follow the inverse operations at insertion (Fig 6). A DWT is applied then, Singular value decomposition will be applied to the three subbands (LL, HL and LH). The Eq. 8 will be applied to extract the watermark bits using the three coefficients LLS, HLS and LHS.

$$
(X=(L L S-\boldsymbol{L H S}) \% 2) \text { and }(Y=(L L S-H L S) \% 2)
$$

An essential part of the extracted bits will be handled to accurately build up the patient details, and the other segment will be used to reconstruct the hash of this specific information. It will then be possible to check the integrity of the extracted data by properly comparing the extracted hash with the hash computed from the patient information.

\section{Experimental results and discussions}

To experiment the proposed approach, the practical tests will be performed on three types of

\begin{tabular}{|c|c|c|c|c|c|c|}
\hline & \multirow{3}{*}{ Apply 2D DWT } & LL & \multirow{3}{*}{$\begin{array}{l}\text { Singular value } \\
\text { decomposition }\end{array}$} & & \multirow[b]{3}{*}{ Extraction process } & \\
\hline & & & & {$[\mathrm{U} \mathrm{S} \mathrm{V}]=\mathrm{SVD}$ (LL) } & & Patient informations \\
\hline \multirow[t]{2}{*}{ Watermarked image } & & & & {$[\mathrm{U} \mathrm{S} \mathrm{V}]=\mathrm{SVD}(\mathrm{HL})$} & & Image informations \\
\hline & & & & {$[\mathrm{U} S \mathrm{~V}]=\mathrm{SVD}(\mathrm{LH})$} & & Hash \\
\hline
\end{tabular}
medical images. The first experiments will be performed on CT images [1]; this dataset was

Fig. 6 Extraction process 

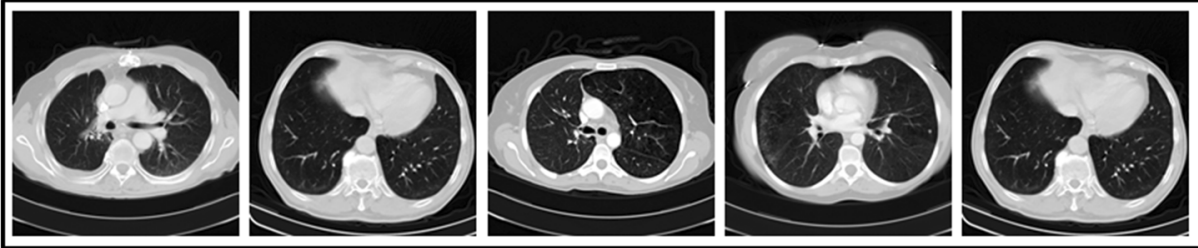

Fig. 7 CT images used for the analysis of the proposed scheme

designed to allow for different methods to be tested for examining the trends in CT image data associated with using contrast and patient age (Fig 7). The data are a tiny subset of images from the cancer imaging archive. They consist of the middle slice of all CT images taken where valid age, modality, and contrast tags could be found. This results in 475 series from 69 different patients.

The second experiments will be performed on X-Ray images. For that, we used the COVID-19 radiographic database [35]. This current release contains 219 COVID-19 positive images, 1341 normal images and 1345 viral pneumonia images. All the images are in Portable Network Graphics (PNG) file format and resolution is 1024-by-1024 pixels (Fig 8), which can be easily converted to 224-by-224 or 227-by-227 pixels typically required by the popular Convolutional Neural Networks (CNNs).

The third experiments will be performed on ultrasound images for this purpose we used an ultrasound images database from the Brno University of Technology [45]. This database contains images of ten volunteers with different weight. Images were acquired with Sonix OP ultrasound scanner with different set-up of depth, gain, time gain compensation (TGC) curve and different linear array transducers (Fig 9). The image database contains 84 B-mode ultrasound images in longitudinal section. The resolution of images is approximately 390x330px.

In order to conduct experiments on natural images, we have also used the FER-2013 dataset [18]. In this database, the data consists of $48 \times 48$ pixel grayscale images of faces (Fig 10). The faces are automatically registered so that the face is more or less centred and occupies about the same amount of space in each image.

The precise measurements of image quality will typically allow us to perform a comparative study of the adaptability of this developed method to these three specific types of images. As we can see in the Fig. 11, the initial and watermarked images are visually similar and the distortions caused by the integration process are visually undetectable. Because of the multiple applications traditionally considered and the various criteria involved, it is delicate to carefully evaluate a watermarking algorithm; however it is possible to correctly identify some essential elements for the objective

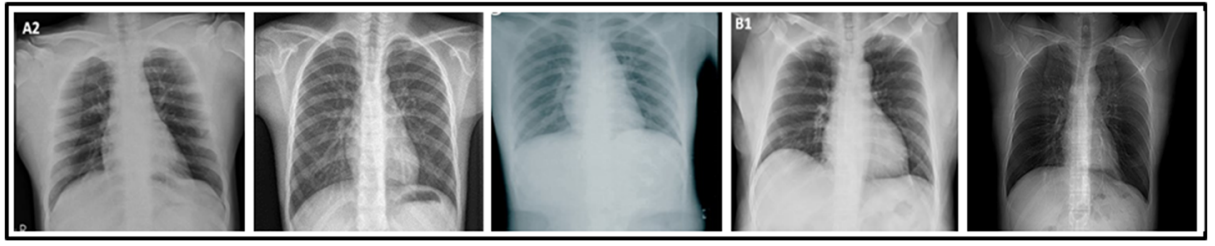

Fig. 8 X-Ray images used for the analysis of the proposed scheme 

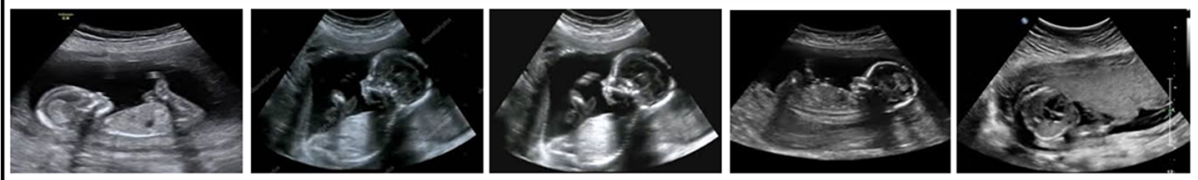

Fig. 9 X-Ray images used for the analysis of the proposed scheme

evaluation of watermarking like the necessary quality of the watermarked image and the robustness of the developed technique against various attacks.

The experimental results are typically based on two distinct stages to properly evaluate our effective approach: the first is dedicated to invisibility, and the second is devoted to robustness against possible attacks.

\subsection{Imperceptibility and capacity test}

To assess the image quality, either objective or subjective measurement is used. Subjective measurements are always expensive, especially if we work with a large sample of medical images. Objective measurements are typically based on a pixel-by-pixel comparison between the initial image and the watermarked image. Objective measurements are based on a pixel-bypixel comparison between the initial image and the watermarked image:

- One of the most popular distortion measures in image processing is the Peak Signal to Noise Ratio (PSNR). The PSNR obtained values between host images (initial images) and watermarked images, in decibels $(\mathrm{dB})$, is typically used to test the considerable distortion introduced by the watermark between these two images [21]. This used ratio is ordinarily used as a necessary measure of the visual quality of the original image relative to the watermarked image. The higher the PSNR, the better the visual quality of the watermarked image. It is traditionally defined by the following formula:

$$
P S N R_{d B}=10 \log _{10}\left[\mathrm{M} * \mathrm{~N} \frac{\max I^{2}(i, j)}{\sum i, j[I(i, j)-J(i, j)]^{2}}\right]
$$

Where $I(i, j)$ is the value of the luminance of the reference pixel $(i, j)$ and $J(i, j)$ that of the image to be tested, the two images being of size $[M \times N]$.
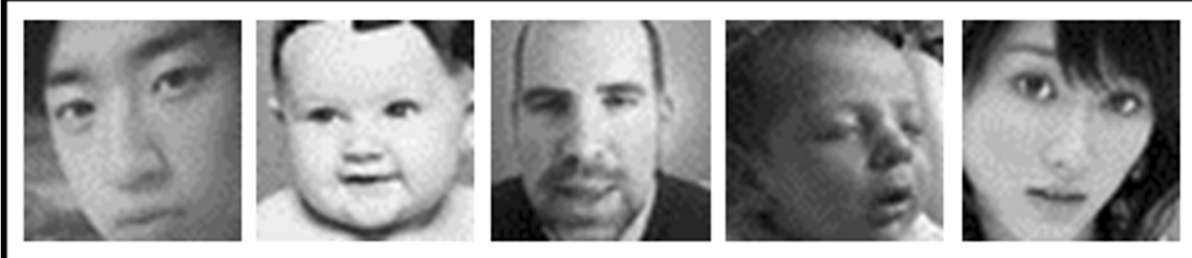

Fig. 10 Natural images used for the analysis of the proposed scheme 


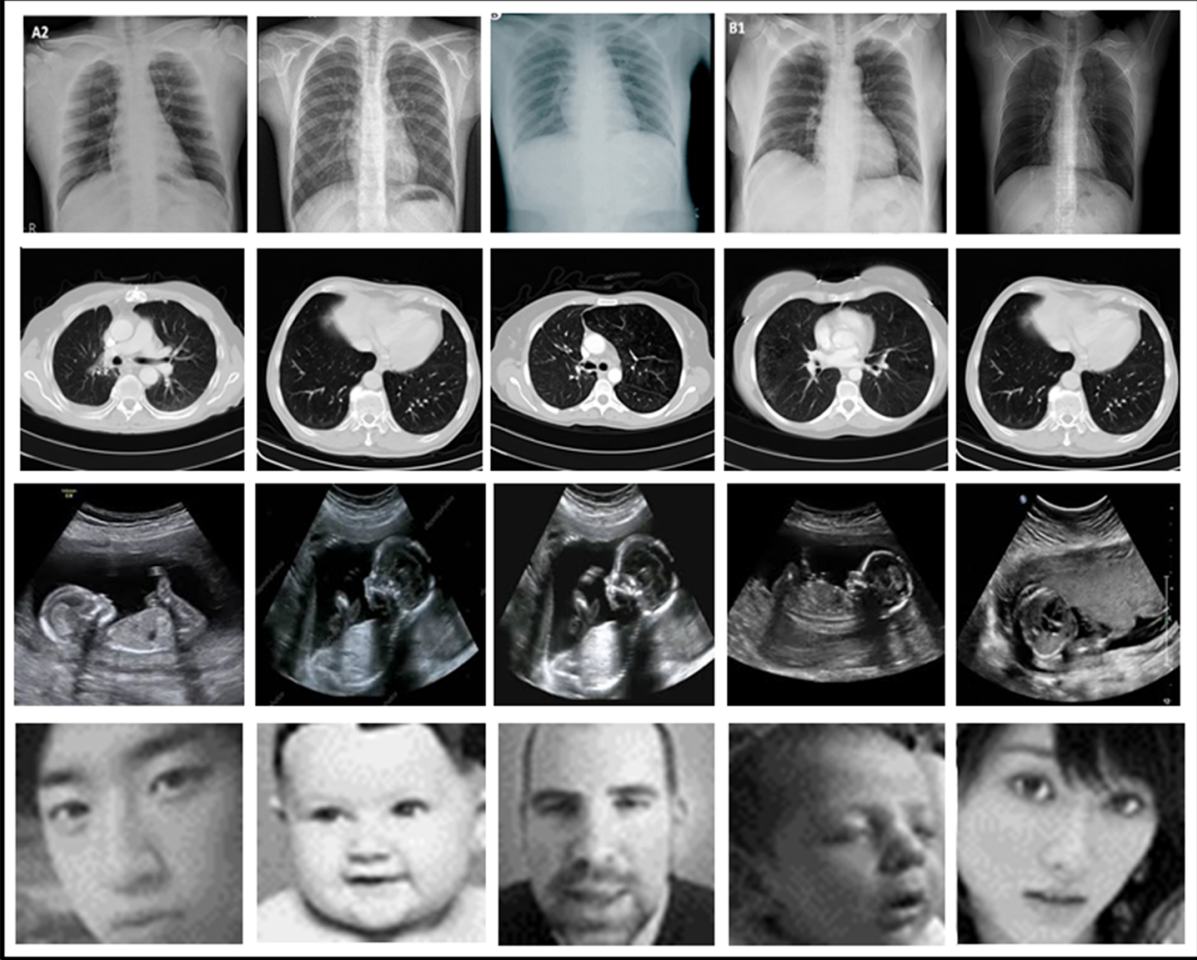

Fig. 11 Watermarked medical images after embedding the watermark contents

- The MSE provides information on the degradation or perceptual distortion introduced at the pixel level [24]. The lower the MSE value, the better the watermarked image is sufficiently appreciated. It is typically defined by the following formula:

$$
\mathrm{MSE}=\frac{1}{M^{*} N} \sum_{i} \sum_{j}(I(i, j)-J(i, j))^{2}
$$

Where I $(i, j)$ is the value of the luminance of the reference pixel $(i, j)$ and $J(i, j)$ that of the image to be tested, the two images being of size [MxN].

- SSIM (Structural SIMilarity) is a measure of essential similarity between two images. It has been developed to measure the visual quality of a compressed image compared to the initial image [34]. The idea of SSIM correctly is to accurately measure the similarity of used structure between the two used images, rather than a pixel-to-pixel difference as is traditionally done for example by PSNR. The SSIM metric is calculated over multiple windows of an image. The precise measurement between two $\mathrm{x}$ and $\mathrm{y}$ windows of size $\mathrm{N} \mathrm{x}$ $\mathrm{N}$ is: 


$$
\operatorname{SSIM}(x, y)=\frac{\left(2 \mu_{x} \mu_{y}+C_{1}\right)\left(2 \delta_{x y}+C_{2}\right)}{\left(\mu_{x}^{2}+\mu_{y}^{2}+C_{1}\right)\left(\delta_{x}^{2}+\delta_{y}^{2}+C_{2}\right)}
$$

Where: $\mu_{x} \quad$ Average of $\mathrm{x}$

$\mu_{y} \quad$ average of $\mathrm{y}$

$\delta_{x}^{2} \quad$ variance of $\mathrm{x}$

$\delta_{y}^{2} \quad$ variance of $\mathrm{y}$

$\delta_{x y} \quad$ covariance of $\mathrm{x} \mathrm{y}$

$C=k 1$ L2 Constant to avoid instability when $\mu_{x}^{2}+\mu_{y}^{2}$ is close to zero $k 1=0.01,=255$.

$C 2=k 2 L 2 \quad$ Constant to avoid instability when $\delta_{x}^{2}+\delta_{y}^{2}$ is close to zero; $k 2=0.03,=255$.

- Number of pixels changed ratio: The NPCR typically indicates the number of pixels modified by the integration process, it is accurately calculated by comparing the pixels of the initial images (CI1) and watermarked image (CI2). The two used images being of size $[\mathrm{M} * \mathrm{~N}]$. The NPCR is precisely defined by the following formula:

$$
\begin{gathered}
\text { NPCR }=\sum_{\mathrm{i}=1}^{\mathrm{M}} \sum_{\mathrm{j}=1}^{\mathrm{N}} \frac{\mathrm{D}(\mathrm{i}, \mathrm{j})}{\mathrm{T}} * 100 \% \\
\mathrm{D}(\mathrm{i}, \mathrm{j})=\left\{\begin{array}{rr}
0, & \text { if } \mathrm{CI}_{1}(\mathrm{i}, \mathrm{j})=\mathrm{CI}_{2}(\mathrm{i}, \mathrm{j}) \\
1, & \text { if } \mathrm{CI}_{1}(\mathrm{i}, \mathrm{j}) \neq \mathrm{CI}_{2}(\mathrm{i}, \mathrm{j})
\end{array}\right.
\end{gathered}
$$

- Bits Per Pixel allow to measure payload capacity, it accurately represents the possible number of bits integrated objectively compared to the image's pixel count.

$$
\mathrm{BPP}=\frac{\text { Number of secret bits embedded }}{\text { Total pixels in the cover image }}
$$

The obtained results in Table 1 show very clearly that our proposed approach offer reasonable imperceptibility while adequately incorporating a large amount of necessary information. The

\begin{tabular}{|c|c|c|c|c|c|}
\hline & PSNR & MSE & SSIM & NPCR & BPP \\
\hline Natural images & 56.37 & 0.1493 & 0.9998 & $49.71 \%$ & 0.4217 \\
\hline CT Images & 55.85 & 0.1830 & 0.9997 & $50.48 \%$ & 0.3974 \\
\hline X-Ray & 57.04 & 0.1251 & 0.9998 & $38.55 \%$ & 0.5442 \\
\hline Ultrasound & 44.92 & 0.3462 & 0.9961 & $69.56 \%$ & 0.3130 \\
\hline
\end{tabular}
successful integration of two bits is achieved by the (eventual) necessary modification of only one out of three coefficients. This reduces the number of necessary modifications performed as

Table 1 Imperceptibility results 
Table 2 Imperceptibility results comparison with related work

\begin{tabular}{llllll}
\hline CT Images & Our & Verma \& al & Gajula \& al & Anand \& al & Memon \& Gilani \\
\hline PSNR & 55.85 & 63.67 & 77.3 & 44.19 & 51.83 \\
X-Ray & Our & Falgun \& al & Assini \& al & Gangadhar \& al & $\begin{array}{l}\text { Thanki \& al } \\
\text { PSNR }\end{array}$ \\
Ultrasound & Our & Badshah \& al & Khor \& al & Alshanbari & Kishore \& al \\
PSNR & 44.91 & 49.61 & 47.824 & 48.37 & 46.34 \\
\hline
\end{tabular}

sufficiently demonstrated by the NPCR rate, which remains low despite the effective integration. This adequately explains the obtained high PSNR rates and the similarity index rate which proves the proposed approach is highly imperceptible. To properly evaluate the imperceptibility of our elaborate scheme, the obtained PSNR rates have been carefully compared to those of the comparable work presented in Section 2.

In order to evaluate the performance of our approach on natural images, we conducted experiments on natural images and compared the rates of PSNR obtained. As we can see in Table 1, the results obtained are almost similar to the results obtained with X-Ray and CT images. As we can see in Table 2, the obtained PSNR rates for the proposed scheme are satisfactory in established relation to recent comparable work. The combination of the parity of the coefficients hides a large amount of data while modifying a reasonable amount of information. This efficiently generates less perceptual distortion and adequately maintains good image quality. In notable addition, since the singular values of the image are stable, adding a slight amount of specific information to the image does not significantly change the singular values. This graciously adequately allows maintaining a good imperceptibility level.

\subsection{Robustness test}

The possible attack is typically defined as any treatment that may subtly alter the mark or cause confusion during its extraction. Several distinct types of attacks can be sufficiently distinguished, which may be intentional or unintentional. Among these classic attacks we consider the addition of noise; Filtering; Lossy compression, mainly JPEG; Geometric transformations (shift, rotation, zoom, slicing...); Analog-to-digital conversion, etc. In the context of medical image watermarking, these images can suffer, particularly, from attacks that can lead to an incorrect diagnosis or an authentication error. To confirm the robustness of our innovative approach against conventional watermarking attacks, we

Table 3 Robustness comparison with related work for CT images watermarking

\begin{tabular}{lllll}
\hline & Our & Verma \& al & Anand \& al & Memon \& Gilani \\
\hline Histogram equalization & 0.8456 & $/$ & 0.7223 & 0.5234 \\
Gaussian noise (0, 0.01) & 0.9811 & 0.9208 & 0.9803 & 0.5273 \\
Sharpening (0.2) & 0.8119 & $/$ & 0.6506 & $/$ \\
Average filtering $(3 \times 1)$ & 0.9732 & 0.838 & 0.986 & 0.4983 \\
Cropping (50\%) & 0.653 & 0.6516 & 0.3586 & $/$ \\
JPEG compression $(30)$ & 0.9631 & $/$ & 0.9388 & 0.4980 \\
Salt \& Pepper Noise (2\%) & 0.9461 & 0.9817 & 0.9251 & $/$ \\
Scaling (50\%) & 0.8406 & 0.7142 & 0.7157 & $/$ \\
\hline
\end{tabular}


Table 4 Robustness comparison with related work for X-Ray images watermarking

\begin{tabular}{lllll}
\hline & Our & Falgun \& al & Assini \& al & Gangadhar \& al \\
\hline Histogram equalization & 0.8056 & 0.9678 & $/$ & 0.9835 \\
Gaussian noise (0, 0.01) & 0.9685 & 0.9632 & 0.9587 & 0.9985 \\
Sharpening (0.2) & 0.8113 & 0.99 & $/$ & $/$ \\
Average filtering $(3 \times 1)$ & 0.9711 & 0.9434 & 0.9909 & 0.9987 \\
Cropping (50\%) & 0.6035 & 0.712 & $/$ & $/$ \\
JPEG compression(30) & 0.9273 & 0.8378 & $/$ & 0.8416 \\
Salt \& Pepper Noise (2\%) & 0.9518 & 0.9657 & $/$ & 0.7476 \\
Scaling (50\%) & 0.838 & 0.9073 & & $/$ \\
\hline
\end{tabular}

properly applied JPEG compression, Salt \& Pepper noise, rotation attack on the watermarked image and some commonly used attacks.

Tables 3, 4 and 5 illustrates obtained performance results in specific terms of NCC. This necessary measure was appropriately applied between the original inserted mark and the extracted mark from the attacked watermarked image. Since the specific Low frequency typically carries most of the valuable information while High frequency includes least valuable information. In our work, for embedding watermark information the low frequency subbands were preferred. It allows us to have a more robust image to attacks like compression where high frequencies in the image are deleted. In addition, a Low Pass Filter does not affect the low frequency components in the image data but should attenuate the high-frequency components. The effective integration of the watermark in the high frequencies will typically imply its possible removal in specific case of image compression or filtering. The results show that even with a significant attack that eliminates a part of the image content, the mark may still properly recovered or carefully extracted with a very satisfactory quality. It is clear that the mark is very well preserved even with the presence of attacks such as compression, noise and cropping.

\subsection{Discussion}

In this work a watermarking approach combining DWT and SVD has been proposed. The considerable advantages of schemes that popularly use the frequency domain as the insertion domain are robust to compression operations since they typically operate the similar domain. On the other hand, the complex calculation of the transform of an image has become less expensive because of the fast transform algorithms. SVD is a crucially important factorization tool in the specific field of image processing, because of its algebraic properties it is more used in digital

Table 5 Robustness comparison with related work for ultrasound images watermarking

\begin{tabular}{lllll}
\hline & Thanki \& al & Our & Alshanbari & Kishore \& al \\
\hline Histogram equalization & 0.9689 & 0.9713 & $/$ & $/$ \\
Gaussian noise (0, 0.01) & 0.7129 & 0.9604 & 0.7941 & 0.6027 \\
Sharpening (0.2) & 0.9408 & 0.9875 & 0.7321 & $/$ \\
Average filtering $(3 \times 1)$ & 0.9538 & 0.9665 & 0.7287 & 0.7892 \\
Cropping (50\%) & 0.7895 & 0.7505 & $/$ & 0.7322 \\
JPEG compression $(30)$ & 0.7374 & 0.8832 & 0.751 & $/$ \\
Salt \& Pepper Noise (2\%) & 0.7054 & 0.9221 & $/$ & $/$ \\
Scaling (50\%) & $/$ & 0.9173 & 0.7704 & \\
\hline
\end{tabular}


watermarking. The used SVD transform typically allows concentrating all the maximum energy of the used image into the minimum singular values. The used SVD transform represents a mathematical tool absolutely necessary in image processing and more used in digital image watermarking which typically produces good results in specific terms of invisibility and robustness. Experimental results have shown this technique provides a satisfactory visual quality of the image and a perfect robustness against some attacks like cropping, JPEG compression and also rotation. Scientifically based on the satisfactory results we can reasonably conclude the proposed approach is more suitable for X-Ray images. This innovative approach typically offers better results in practical terms of imperceptibility and robustness compared to recent comparable work.

However, this approach suffers from the DWT poor directional information and the SVD complexity. Effectively, DWT is a typical multi-scale transform (MST) method that does not have the translation-invariance property. In addition, DWT decompose the image into one lowfrequency sub-band and three high-frequency sub-bands (horizontal, vertical, and diagonal). Only three decomposition directions are not enough to represent textures of different scales. To fix this problem it would be interesting in our future work, to use non-subsampled MST methods, such as non-subsampled contourlet transform (NSCT) or non-subsampled shearlet transform (NSST). In addition, Schur decomposition could be an alternative to the singular values decomposition. Singular value decomposition requires about $11 \mathrm{~N}^{3}$ flops as compared to a Schur decomposition which requires only $8 \mathrm{~N}^{3} / 3$. In order to reduce computation time and complexity, it would be advantageous to use schur decomposition instead of singular value decomposition.

However, in the proposed approach the watermark is not encrypted. To increase the watermark's security, an encryption can be added before the integration process. For this, two options will be possible. The first one will be to use a public key infrastructure, in this approach; each doctor will have a public and a private key. The doctor will then sign the image with his private key; this will guarantee the integrity of the watermark and can be verified by all those who have access to the infrastructure using the doctor's public key. The second approach will consist in generating a symmetric key before each transfer, the watermark will then be encrypted with this key by the sender and the receiver (holder of the same key) will be able to decrypt the watermark. As this key is similar for both responsible parties, symmetrical encryption could be properly used to take advantage of its reasonable speed (about eight times faster than asymmetrical encryption).

\section{Conclusion}

To enhance the security of data exchanged in telemedicine, we had proposed in this work a watermarking approach combining DWT and SVD. The proposed approach protects the images exchanged in telemedicine but also avoids any confusion between patient records (even at the local level). As we can see nowadays, medical samples are transmitted to specialized centers. The data collected from patients must be handled with care, in fact, it is necessary to avoid confusing two different radiographies of two different patients; confusion in such a pandemic could have serious consequences for the patient. For this reason the watermark provides a satisfactory solution, since as soon as the patient's information is acquired, it is integrated into the image. Even after the image has been transferred or stored, the doctor will be able to state with certainty that the image really belongs to the patient being treated. The proposed approach has been experimented at a local level; the implementation of such an approach in a medical network for a telemedicine infrastructure will therefore be easy 
and inexpensive. It will just consist in integrating a module to the image acquisition and storage system, in order to watermark the image just after its acquisition. The goal is thus to protect it even at the local level.

\section{Declarations}

Ethical approval This article does not contain any studies with human participants or animals performed by any of the authors.

Conflict of interest The authors declare that they have no known competing financial interests or personal relationships that could have appeared to influence the work reported in this paper.

\section{References}

1. Albertina B, Watson M, Holback C, Jarosz R, Kirk S, Lee Y Lemmerman J (2016) Radiology data from the Cancer genome atlas lung adenocarcinoma collection. The Cancer Imaging Archive. https://doi.org/10. 7937/K9/TCIA.2016.JGNIHEP5

2. Alshanbari S (2020) Medical image watermarking for ownership \& tamper detection, Multimed Tools Appl, https://doi.org/10.1007/s11042-020-08814-9

3. Anand A, Kumar Singh A (2020) An improved DWT-SVD domain watermarking for medical information security. Comput Commun 152:72-80

4. Aparna P, Kishore P (2019) Biometric-based efficient medical image watermarking in E-healthcare application. IET Image Process 13:421-428

5. Assini I, Badri A, Safi K, Sahel A, Baghdad A (2018) A robust hybrid watermarking technique for securing medical image. Int J Intell. Eng Syst 11:169-176. https://doi.org/10.22266/ijies2018.0630.18

6. Badshah G, Liew S, Zain J, Ali M (2016) Watermarking of ultrasound medical images in teleradiology using compressed watermark. J Med Imaging 3:1-9

7. Barania M, Peyman A, Valandar M, Irani B (2020) A blind video watermarking algorithm robust to lossy video compression attacks based on generalized Newton complex map and contourlet transform. Multimed Tools Appl 79:2127-2159

8. Barani MJ, Ayubi P, Yousefi Valandar M, Irani BY (2020) A new Pseudo random number generator based on generalized Newton complex map with dynamic key. J Inf Secur Appl 53:102509. https://doi.org/10. 1016/j.jisa.2020.102509

9. Cedillo-Hernandez M, Cedillo-Hernandez A, Nakano-Miyatake M, Perez-Meana H (2020) Improving the management of medical imaging by using robust and secure dual watermarking. Biomed Signal Process Control 56:101695. https://doi.org/10.1016/j.bspc.2019.101695

10. Degadwala D, Kulkarni M, Vyas D, Mahajan A (2020) Novel image watermarking approach against noise and RST attacks. Procedia Comput Sci 167:213-223

11. Devi H, Singh K (2020) Red-cyan anaglyph image watermarking using DWT, Hadamard transform and singular value decomposition for copyright protection, J Inf Secur Appl, Volume 50

12. Du L, Ho ATS, Cong R (2020) Perceptual hashing for image authentication: a survey. Signal Process Image Commun 81:115713. https://doi.org/10.1016/j.image.2019.115713

13. Euschi S, Khaldi A, Kafi R, Kahlessenane F (2021) A Fourier transform based audio watermarking algorithm, applied acoustics. Volume 172:107652. https://doi.org/10.1016/j.apacoust.2020.107652

14. Fan T, Chao H, Chieu B (2019) Lossless medical image watermarking method based on significant difference of cellular automata transform coefficient. Signal Process Image Commun 70:174-183

15. Favorskaya M, Savchina E, Gusev K (2019) Feature-based synchronization correction for multilevel watermarking of medical images. Procedia Comput Sci 159:1267-1276

16. Gajula S, Rajesh V (2017) Medical image watermarking scheme with DWT \& SVD transforms. Int J Pure Appl Math 117:285-290

17. Gangadhar Y, Giridhar Akula VS, Chenna Reddy P (2018) An evolutionary programming approach for securing medical images using watermarking scheme in invariant discrete wavelet transformation. Biomed Signal Process Control 43:31-40

18. Goodfellow IJ, Erhan D, Carrier PL, Courville A, Mirza M, Hamner B, Cukierski W, Tang Y, Thaler D, Lee D-H, Zhou Y, Ramaiah C, Feng F, Li R, Wang X, Athanasakis D, Shawe-Taylor J, Milakov M, Park J, 
Ionescu R, Popescu M, Grozea C, Bergstra J, Xie J, Romaszko L, Xu B, Chuang Z, Bengio Y (2015) Challenges inrepresentation learning: A report on three machine learning contests. Neural Netw 64:59-63

19. Haddad S, Coatrieux G, Cozic M, Bouslimi D (August 2017) Joint watermarking and lossless JPEG-LS compression for medical image security. IRBM 38(4):198-206

20. Haddad S, Coatrieux G, Moreau-Gaudry A, Cozic M (February 2020) Joint watermarking-encryptionJPEG-LS for medical image reliability control in encrypted and compressed domains. IEEE Trans Inf Forensics Secur 15:2556-2569

21. Hashim M, Rahim M, Johi F, Taha M, Hamad H (2018) Performance evaluation measurement of image steganography techniques with analysis of LSB based on variation image formats. Int J Eng Technol 7: 3505-3514

22. Hassan B, Ahmed R, Li B, Hassan O (2019) An imperceptible medical image watermarking framework for automated diagnosis of retinal pathologies in an eHealth arrangement. IEEE Access 7:69758-69775

23. Hu H, Hsu L, Chou H (2020) An improved SVD-based blind color image watermarking algorithm with mixed modulation incorporated. Inf Sci 519:161-182

24. Kahlessenane F, Khaldi A, Euschi S (2020) A robust blind color image watermarking based on Fourier transform domain, Optik, Volume 208

25. Kahlessenane F, Khaldi A, Kafi R, Euschi S, A DWT based watermarking approach for medical image protection, J Ambient Intell Humaniz Comput, (2020). https://doi.org/10.1007/s12652-020-02450-9

26. Khaldi A (2018) Diffie-Hellman key exchange through Steganographied images. Revista de Direito Estado e Telecomunicações 10:147-160

27. Khaldi A (2019) Steganographic techniques classification according to image format. Int Ann Sci 8:143149

28. Khaldi A (2020) A lossless blind image data hiding scheme for semi-fragile image watermark. Int J Comput Vis Robot 10:373. https://doi.org/10.1504/IJCVR.2020.10029218

29. Khor HL, Liew S, Zain J (2016) Parallel digital watermarking process on ultrasound medical images in multicores environment. Int J Biomed Imaging 2016:1-14

30. Kishore PVV, Venkatram N, Sarvya C, Reddy LSS (2014) Medical image watermarking using RSA encryption in wavelet domain, In 2014 First International Conference on Networks Soft Computing (ICNSC2014), pp. 258-262, doi: https://doi.org/10.1109/CNSC.2014.6906662.

31. Koohpayeh Araghi T, Abd Manaf A (2019) An enhanced hybrid image watermarking scheme for security of medical and non-medical images based on DWT and 2-D SVD. Futur Gener Comput Syst 101:12231246

32. Liu X, Lin C, Yuan S (2018) Blind dual watermarking for color images' authentication and copyright protection. IEEE Trans Circuits Syst Video Technol 28:1047-1055. https://doi.org/10.1109/TCSVT.2016. 2633878

33. Memon NA, Gilani SAM, Ali A (2009) Watermarking of chest CT scan medical images for content authentication, In 2009 International Conference on Information and Communication Technologies, pp. 175-180, doi: https://doi.org/10.1109/ICICT.2009.5268167.

34. Mun S, Nam S, Jang H, Kim D, Lee H (2019) Finding robust domain from attacks: a learning framework for blind watermarking. Neurocomputing, Volume 337(14):191-202

35. Paul Cohen J, Morrison P, Dao L (2020) COVID-19 image data collection. https://github.com/ieee8023/ covid-chestxray-dataset

36. Peyman A, Setayeshi S, Rahmani A (2020) Deterministic chaos game: a new fractal based pseudo-random number generator and its cryptographic application, J Inf Secur Appl Volume 52

37. Qasim F, Meziane F, Aspin R (2018) Digital watermarking: applicability for developing trust in medical imaging workflows state of the art review. Comput Sci Rev 27:45-60

38. Sakthidasan K, Abhi Rayna H, Mangu V, Prakash VR, Vasudevan N (2019) Image water marking using DWT to encapsulate data in medical image, international conference on communication and signal processing, India

39. Sankaran KS, Rayna HA, Mangu V, Prakash VR, Vasudevan N (2019) Image water marking using DWT to encapsulate data in medical image. In: 2019 presented at the 2019 International Conference on Communication and Signal Processing (ICCSP), pp 0568-0571. https://doi.org/10.1109/ICCSP.2019. 8698057

40. Thakkar FN, Srivastava VK (2017) A blind medical image watermarking: DWT-SVD based robust and secure approach for telemedicine applications. Multimed Tools Appl 76:3669-3697. https://doi.org/10. 1007/s1 1042-016-3928-7

41. Thanki R, Borra S (2019) Fragile watermarking for copyright authentication and tamper detection of medical images using compressive sensing (CS) based encryption and contourlet domain processing. Multimed Tools Appl 78:13905-13924 13905 
42. Thanki R, Borra S, Dwivedi V, Borisagar K (Aug. 2017) An efficient medical image watermarking scheme based on FDCuT-DCT. Eng Sci Technol Int J 20(4):1366-1379. https://doi.org/10.1016/j.jestch.2017.06. 001

43. Verma U, Sharma N (2019) Hybrid mode of medical image watermarking to enhance robustness and imperceptibility. Int J Innovative Technol Exploring Eng 9:351-359

44. Zhang L, Wei D (2020) Image watermarking based on matrix decomposition and gyrator transform in invariant integer wavelet domain, Signal Process Volume 169

45. Zukal M, Beneš R, Č́́ka P, ̌̌íha K (2011) Ultrasound image database. Department of Telecommunications, Brno University of Technology. http://splab.cz/en/download/databaze/ultrasound

Publisher's note Springer Nature remains neutral with regard to jurisdictional claims in published maps and institutional affiliations.

\section{Affiliations}

\section{Narima Zermi ${ }^{1} \cdot$ Amine Khaldi $^{2} \cdot$ Med Redouane Kafi $^{2} \cdot$ Fares Kahlessenane $^{2} \cdot$ Salah Euschi $^{2}$}

1 Electronics Department, Faculty of Engineering Sciences Laboratories of Automation and Signals of Annaba (LASA), Badji Mokhtar Annaba University, 23000 Annaba, Algeria

2 Computer Science Department, Faculty of Sciences and Technology, Artificial Intelligence and Information Technology Laboratory (LINATI), University of Kasdi Merbah, 30000 Ouargla, Algeria 